\title{
A 5-YEAR EXPERIENCE OF A MINIMALLY INVASIVE TECHNIQUE FOR CORRECTION OF PECTUS EXCAVATUM IN CROATIA
}

\author{
Mirko Žganjer, Božidar Župančić, Ljiljana Popović
}

Children's Hospital Zagreb, Croatia: Department of Pediatric surgery

Summary: The aim of this study was to assess the results of 5-year experience with minimally invasive operation without medial incision and resection cartilages for correction of pectum excavatum. From 2000 we made in our Hospital minimally invasive technique for the correction of pectus excavatum. 75 patients were treated by minimally invasive technique. A convex steel bar is inserted under the sternumtrough small bilateral Incisions. The steel bar is inserted with the convexity facing posteriorly, and when it is in position, the bar is turned over. After 2 years the bar is removed when permanent remolding has occurred. Initial excelent results were maintained in 54 patients (normal chest), good results in 16 (mild residual pectus) and poor in 5 (severe recurrence requiring further treatment). The mean follow-up since surgery were 3 months to 3 years. Average blood loss was $25 \mathrm{ml}$. Average length of hospital stay was 8 days. Patients returned to full activity after 2 month. Complications were pneumothorax in 12 patients, pneumonia in 6 patients and displacement of the steel barr requiring revision in 2 patients. Poor results occurred because steel bar was too soft in 3 patients, and soft sternum in 2 patients with Marfan's syndrome. The minimally invesive technique is effective without cartilage incision and resection or sternal osteotomy.

Key words: Pectum excavatum, Minimally invasive surgery, Children

\section{Introduction}

The aim of this study was to assess the results of 5-year experience with minimally invasive operation without medial incision and resection cartilages for correction of pectum excavatum.

From 2000 we made in our Hospital minimally invasive technique for the correction of pectus excavatum. 75 patients were treated by minimally invasive technique. A convex steel bar is inserted under the sternum trough small bilateral Incisions. The steel bar is inserted with the convexity facing posteriorly, and when it is in position, the bar is turned over. After 2 years the bar is removed when permanent remolding has occurred.

Initial excelent results were maintained in 54 patients (normal chest), good results in 16 (mild residual pectus) and poor in 5 (severe recurrence requiring further treatment). The mean follow-up since surgery were 3 months to 3 years. Average blood loss was $25 \mathrm{ml}$. Average length of hospital stay was 8 days. Patients returned to full activity after 2 month. Complications were pneumothorax in 12 patients, pneumonia in 6 patients and displacement of the steel barr requiring revision in 2 patients. Poor results occurred because steel bar was too soft in 3 patients, and soft sternum in 2 patients with Marfan's syndrome.
The minimally invesive technique is effective without cartilage incision and resection or sternal osteotomy.

\section{Materials and methods}

When we have a children with deformity of the chest wall it should be possible to remodel the chest wall. In children whose ribs and cartilages are still soft it is not make cartilage incisions, resections or sternal osteotomy. By inserting a convex steel bar under the sternum trough a small lateral thoracic incisions without cartilage resections or sternum osteotomy, it was possible to correct even a severe degree of pectus excavatum (3). This report presents our experience with minimally invasive procedure over a 5-year period.

From 2000 to 2005,75 patients with pectus excavatum had minimally invasive surgery. In the pectus excavatum group were 50 boys and 25 girls and sex ratio of 3:1.

All pectus excavatum patients underwent an exercise and posture program and preoperative follow-up at 6 month intervals.

Indications for reconstructive surgery based in 42 patients $(56 \%)$ on the severity of the defects. Other patients had clinical signs and symptoms are listed in Tab. 1 (clinical signs and symptoms). The ages of the patients at the 
time of surgery varied from 7 to 20 years and the maximum number of patients were in the 12 to 14 year age group $(4,6)$.

In all patients underwent history evaluation and physical examination, chest $\mathrm{X}$ ray, complete blood count, PT, PTT and urinalysis. In all patients we make cardiology and pulmology examinations. Computed tomography (CT) scans were performed only in 2 patients when we want to document the severity of the deformity (8).

\section{Surgical Technique}

Before 2000 our technique included anterior thoracic incision,resection of cartilage and insertion steel bar (5). Since 2000 steel bar has been inserted through a lateral thoracic incision without sternal osteotomy and cartilage resections. Before operative surgery patient's chest was measured and correct length steel bar selected and bent with bar bender. One day before operative procedure all pa-

Tab. 1: Clinical signs and symptoms.

\begin{tabular}{|l|c|}
\hline Symptom & No.of patients \\
\hline Exercise intolerance and dyspnea & 21 \\
\hline Chest pain with exercise & 9 \\
\hline Arrhythmia & 8 \\
\hline Asthma & 2 \\
\hline Marfan's syndrome & 2 \\
\hline
\end{tabular}

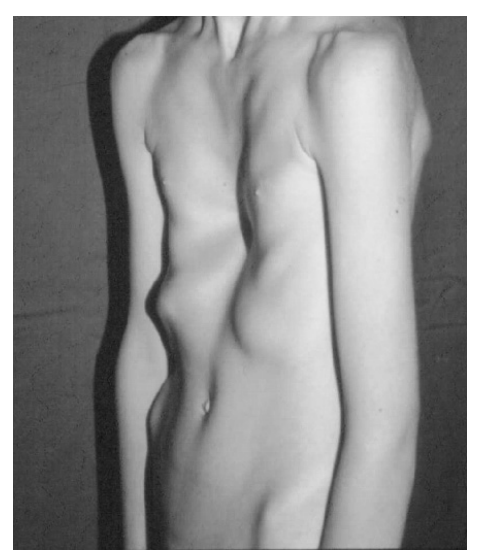

Fig. 1: Pectus excavatum.

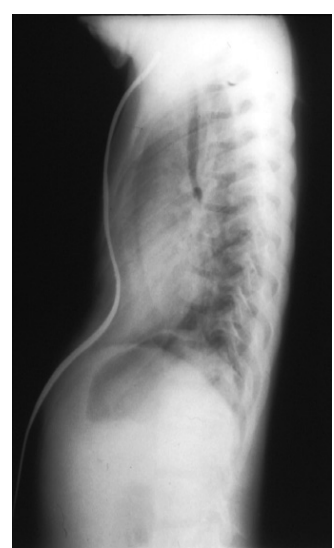

Fig. 2: Chest X-ray.

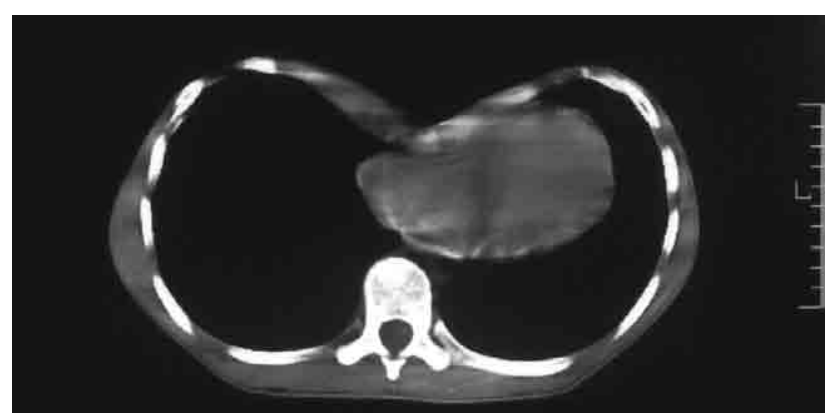

Fig. 3: CT scan. tients were given cefazolin. The operation was performed under endotracheal anestesia and epidural block (for postoperative pain control). The patient was positioned with both arms abducted. When patient was in good position the previosly steel bar bent into its convex shape to confirm anterior chest wall curvature. A transverse incisions was made in each lateral chest wall in middle axillary lines. A skin tunnel was raised anteriorly and entered with curved Kelly clamp through the mediastinum under the sternum until it emerged on the opposite side.

Two strands of umbilical tape were pulled through the mediastinum. One strand was then used to guide for previosly prepared steel bar and also steel bar was pulled with tape. The bar was passed under the sternum with the convexity facing posteriorly. When the bar was in position, it was turned over and raising sternum and anterior chest wall into new and good position. We never placed two bars in the same patient. The bar was secured with stabilizer and haevy sutures to the lateral chest wall muscles. Before closing the incisions, positive expiratory pressure $(4$ to $5 \mathrm{~cm}$ $\mathrm{H} 20$ ) was added to prevent pleural air traping. The wounds closed in layers. The chest radiogram was obtained to check for pneumothorax. For the first few days patients kept well sedated to prevent dispalcement of the bar. Regular activity was permitted after 2 months. Two years after operative treatement the bar removed under general anestesia.

\section{Results}

Of 75 patients who had minimally invasive operations 9 patients were under 10 year old, 28 patients were between 10 and 14 years old, 37 patients were between 14 and 16 years old. One patient was 20 year old. The length of hospitalization varied from 6 to 15 days (average 8 days). Blood loss was minimal, average $25 \mathrm{ml}$. No minimally invasive procedure patient required transfusion.

Complications in 75 minimally invasive procedure patients included 12 cases of pneumothorax. In 8 patients pneumothorax was small and resolved spontaneously. In 4 patients tube thoracostomy was responded. Postoperative viral pneumonia developed in 6 patients and that patients responded to supportive care. In 3 patients steel bar was too soft and it is necessary to remove steel bar and reimplantation second. In 2 patients with Marfan's syndrome sternum was not strong enough and collapsed above and below the metal bar. It was necessary to reoperation by cartilage resection and sternal osteotomy $(1,7)$.

\section{Discussion}

Since 2000 minimally invasive procedure is standard in our hospital for treatment pectus excavatum. Before 2000 we used cartilage resections, sternal osteotomy and fixation of the sternum in normal position. Standard procedure are also successfully but operating time is longer, increased 
blood loss, ribs growth is sometimes impaired, scars over thorax is visible and duration of hospitalization is longer. The advantages of this minimally invasive procedure are: 1) no anterior chest wall incision 2) the scars are unsightly 3) short operating time 4) minimal blood loss 5) early return to normal activity. In our Hospital we have not long term results but we hope that our results will be excellent $(2,5)$.

\section{References}

1. Arn PH, Scherer LR, Haller JA Jr., Pyeritz RE: outcome of pectus excavatum with Marfan syndrome in general population. Jourenal of Pediatrics, 1989;115 230

2. Croitoru DP, Kelly RE, Goretsky MJ, Lawson ML, Swoveland B, Nuss D: Experience and modification update for the minimally invasive Nuss technique for pectus excavatum repair in 303 patients, Journal of Pediatric Surgery, 2002; 37:437.

3. Fonkalsrud EW: Current management of pectum excavatum, World Journal of Surgery, 2003;27:502.

4. Haller JA Jr: Severe chest wall construction from growth retardation after too extensive and too early ( $<4$ years) pectus excavatum repair, Annals of Thoracic Surgery, 1995;60:88.

5. Hosie S, Sitkiewitz T, Petersen C, Gobel P, Schaarschmidt K, Till H: Minimally invasive repair of pectus excavatum-the Nuss procedure. A European multicentre experience, European Journal of Pediatric Surgery, 2002;12:235.

6. Nuss D, Croitoru DP, Kelly RE Jr., Goretsky MJ, Nuss KJ, Gustin TS: review and discussion of the complications of minimally invasive pectus exvacatum repair European Journal of Pediatric Surgery, 2002;12:230.

7. Park HJ, Lee CS, Youm W, Lee KR. The Nuss procedure for pectus excavatum: evolution of techniques and early results on 322 patients, Annals of Thoracic Surgery, 2004;77:289.

8. Sigalet DL, Montgomery M, Harder J: Pulmonary function and excersise response in patients with pectus excavatum after Nuss repair, Journal of Pediatric Surgery, 2003;38:544

Submitted September 2005.

Accepted April 2006.

Mirko Žganjer, M.D., Children's Hospital Zagreb, Department of Pediatric surgery,

Klaićeva 16, 10000 Zagreb,

Croatia. e-mail: mirko.zganjer@zg.htnet.hr 